\section{Antibiotic resistance genes in the environment: prioritizing risks}

\author{
Johan Bengtsson-Palme and D. G. Joakim Larsson
}

In a recent Opinion article (What is a resistance gene? Ranking risks in resistomes. Nature Rev. Microbiol. 13, 116-123 (2015)) ${ }^{1}$, Martínez et al. outline the obstacles associated with determining the risks presented by antibiotic resistance genes in environmental microbial communities in terms of their potential to transfer to human pathogens. The authors then propose a system (resistance readiness condition (RESCon)) for ranking the risks associated with the detection of such genes. Within this system, antibiotic resistance genes that are already present on mobile genetic elements in human pathogens are ascribed the highest risk (RESCon 1), whereas resistance genes encoding novel resistance mechanisms that have not yet been found on mobile elements in a pathogen are considered to be part of lower risk categories. We believe that the proposed assessment scheme overestimates the risks associated with well-known resistance genes that are already circulating among human pathogens and underappreciates the potential consequences of the transfer of previously unknown resistance determinants from the environmental resistome.

Within a risk-assessment framework, two concepts are fundamental: the probability of an event occuring and the severity of the consequences of such an event. In the context discussed here, the event involves the transfer of a functional resistance gene from environmental bacteria to a pathogen, which then has the potential to infect patients and result in treatment failure. We argue that the transfer of well-known resistance genes from the external environment is probably rare and is also unlikely to dramatically alter treatment outcomes, as many pathogens already carry these genes. In comparison, the transfer of such genes between pathogens (or from human commensals to pathogens) would be expected to occur much more frequently in the human gut, as the most crucial players are often already present at this site (that is, the mobilized resistance gene, the receiving pathogen and the selective pressure for transfer). It is therefore difficult to envisage a scenario in which known resistance genes in the external environment have a central role in the emergence of multi-resistant pathogens. By contrast, we argue that the presence of novel environmental resistance determinants is likely to present a much greater risk, particularly if these genes encode novel resistance mechanisms or confer resistance to antibiotics that are under development, widely used or last-resort antibiotics ${ }^{1,2}$. The reason is simply that there are much greater potential consequences involved, despite a possibly lower probability of transfer. Although the origin of the gene encoding New Delhi metallo- $\beta$-lactamase (NDM1) is unknown, its emergence in a clinical context and its rapid transfer to several pathogens on a global scale exemplify such a scenario ${ }^{3,4}$.

Martínez et al. stress that although resistance genes are found everywhere, all environments do not pose the same risk. We strongly agree, but we propose that the abundance and diversity of resistance genes also need to be considered to evaluate the associated risks; high abundance and diversity are likely to be associated with a higher probability of transfer, simply owing to the increased likelihood for potential donor strains to physically interact with suitable receivers ${ }^{5,6}$. In addition, high abundance can - but does not need to be - a reflection of a selective pressure that favours mobilization. It is important to recall that, in principle, resistance need only emerge once in a single cell in order for it to have potential to rise in frequency and be transferred ${ }^{7}$. Thus, it is crucial to identify environmental hotspots where novel resistance genes could emerge, and we should subsequently reduce selective pressures, transfer and dissemination opportunities at such sites. For example, such hotspots have been identified in environments that are subjected to pollution by waste from antibiotic-production factilities ${ }^{8-10}$.

We acknowledge that the fitness costs associated with resistance genes, as well as their amelioration owing to compensatory mutations, are often neglected, and that this may prevent many resistance genes from becoming a clinical problem. Although we disagree that the prior presence of resistance genes in pathogens would necessarily equate to a higher risk, as argued by Martínez et al., we fully agree with the authors' assessment of risk being determined by several factors, such as the demonstrated mobility of the gene, whether its function is demonstrated or only predicted, and whether the environment in question provides transmission routes for resistant bacteria to transfer to humans or domestic animals ${ }^{1,7,11-13}$. Assessing risks that are associated with environmental resistomes is indeed non-trivial: the detection of resistance genes may reflect a selective pressure enriching for such genes among resident bacteria; contamination with bacteria of human or animal origin (including faecal bacteria) that became resistant during therapy and not in the environment; or a combination of both. The origin of detected resistance genes and the absence or presence of a selective pressure have major implications for managing risks. Future studies should therefore go beyond simply quantifying resistance genes to assess their genetic context, their potential for horizontal gene transfer, their compatibility with putative pathogenic hosts and the prevailing selective pressure. Taken together, the proposed RESCon scheme by Martínez et al. is likely to correspond reasonably well to the probability that transfer will occur, but we believe that it does not sufficiently account for the possible consequences of such transfer events, which we argue are greatest in those cases in which the mobilized resistance gene has not yet been detected in pathogenic bacteria.

Johan Bengtsson-Palme and D. G. Joakim Larsson
are at the Department of Infectious Diseases,
Institute of Biomedicine, Sahlgrenska Academy,
University of Gothenburg, Guldhedsgatan 10A,
SE-413 46, Gothenburg, Sweden
Correspondence to D.G.J.L.
e-mail: joakim.larsson@fysiologi.gu.se
doi:10.1038/nrmicro3399-c1
Published online 27 April 2015

1. Martínez, J. L., Coque, T. M. \& Baquero, F. What is a resistance gene? Ranking risk in resistomes. Nature Rev. Microbiol. 13, 116-123 (2015).

2. Gaze, W. H. et al. Influence of humans on evolution and mobilization of environmental antibiotic resistome. Emerging Infect. Dis. 19, 4 (2013). Kumarasamy, K. K. et al. Emergence of a new antibiotic resistance mechanism in India, Pakistan, and the UK: a molecular, biological, and epidemiological study. Lancet Infect. Dis. 10, 597-602 (2010)

4. Walsh, T. R., Weeks, J., Livermore, D. M. \& Toleman, M. A. Dissemination of NDM-1 positive bacteria in the New Delhi environment and its implications for human health: an environmental point prevalence study. Lancet Infect. Dis. 11, 355-362 (2011).

5. Levin, B. R., Stewart, F. M. \& Rice, V. A. The kinetics of conjugative plasmid transmission: fit of a simple mass action model. Plasmid 2, 247-260 (1979).

6. Fernandez-Astorga, A., Muela, A., Cisterna, R., Iriberri, J. \& Barcina, I. Biotic and abiotic factors affecting plasmid transfer in Escherichia coli strains. Appl. Environ. Microbiol. 58, 392-398 (1992). 
7. Ashbolt, N. J. et al. Human health risk assessment (HHRA) for environmental development and transfer of antibiotic resistance. Environ. Health Perspect. 121, 993-1001 (2013).

8. Kristiansson, E. et al. Pyrosequencing of antibioticcontaminated river sediments reveals high levels of resistance and gene transfer elements. PLOS ONE 6, e17038 (2011).

9. Bengtsson-Palme, J., Boulund, F. Fick, J. Kristiansson, E. \& Larsson, D. G. J. Shotgun metagenomics reveals a wide array of antibiotic resistance genes and mobile elements in a polluted lake in India. Front. Microbiol. 5, 648 (2014)

10. Marathe, N. P. et al. A treatment plant receiving waste water from multiple bulk drug manufacturers is a reservoir for highly multi-drug resistant integron-bearing bacteria. PLOS ONE 8, e77310 (2013).

11. Wellington, E. M. et al. The role of the natural environment in the emergence of antibiotic resistance in Gram-negative bacteria. Lancet Infect. Dis. 13 . 155-165 (2013).
12. Dantas, G. $\&$ Sommer, M. O. Context matters - the complex interplay between resistome genotypes and resistance phenotypes. Curr. Opin. Microbiol. 15 577-582 (2012).

13. Martinez, J. L. Bottlenecks in the transferability of antibiotic resistance from natural ecosystems to human bacterial pathogens. Front. Microbiol. 2, 265 (2011).

Competing interests statement

The authors declare no competing interests. 